\title{
Reflets
}

Revue ontaroise d'intervention sociale et communautaire

\section{Au service de la jeunesse : le programme ontarien l'Avenir}

\section{Maxim Gravel}

Volume 3, numéro 1, printemps 1997

Enfance et familles en contexte d'appauvrissement

URI : https://id.erudit.org/iderudit/026156ar

DOI : https://doi.org/10.7202/026156ar

Aller au sommaire du numéro

Éditeur(s)

Reflets : Revue ontaroise d'intervention sociale et communautaire

ISSN

1203-4576 (imprimé)

1712-8498 (numérique)

Découvrir la revue

Citer cet article

Gravel, M. (1997). Au service de la jeunesse : le programme ontarien l'Avenir. Reflets, 3(1), 138-142. https://doi.org/10.7202/026156ar

Tous droits réservés (C) Reflets : Revue ontaroise d'intervention sociale et communautaire, 1997
Ce document est protégé par la loi sur le droit d'auteur. L'utilisation des services d'Érudit (y compris la reproduction) est assujettie à sa politique d'utilisation que vous pouvez consulter en ligne.

https://apropos.erudit.org/fr/usagers/politique-dutilisation/ 


\title{
Au service de la jeunesse : le programme ontarien 1'Avenir
}

\author{
Maxim Gravel \\ Collège Northern, Kirkland Lake
}

La lutte contre la pauvreté et tous les problèmes qu'elle engendre doit inclure, entre autres, une variété de stratégies pour permettre aux personnes de s'approprier les moyens de s'en sortir. L'Avenir, un programme de formation d'emploi conçu en Ontario en 1985, a pour but de soutenir le cheminement des jeunes de 16 à 24 ans vers une pleine participation au marché du travail.

\section{La nécessité d'un programme de formation pour les jeunes}

Lorsqu'on constate dans le Rapport sur le marché du travail de mai 1996 du Conseil ontarien de formation et d'adaptation de la main-d'oeuvre que le taux de chômage chez les 15 à 19 ans est de $19,1 \%$ et de $14,9 \%$ pour les 20 à 24 ans tandis que le taux de chômage provincial est de 9,3\%, nous mettons en évidence l'existence d'une inégalité, selon l'âge, de la distribution du travail à la main d'oeuvre de l'Ontario. Quoique le but de cet article ne soit pas de faire une analyse exhaustive des facteurs expliquant cette inéquité, il offre une toile de fond pour mieux comprendre certaines dimensions des nombreux obstacles auxquels se butent les jeunes à la recherche d'un emploi.

Dans le contexte économique actuel, le déplacement des travailleurs et des travailleuses est monnaie courante et malheureusement, les jeunes doivent faire concurrence à des chômeurs et des chômeuses qui bénéficient d'une plus vaste expérience de travail pour négocier les emplois disponibles. Plusieurs se 
retrouvent donc dans une situation où ils et elles ont besoin d'expérience de travail pour décrocher un emploi mais ne peuvent pas en accumuler faute d'accès aux emplois disponibles! Le programme l'Avenir intervient pour eux dans ce cercle vicieux.

\section{Modalités du programme}

Les jeunes sans-emploi sont invités à se présenter à des séances d'information dans le but de se familiariser avec les objectifs du programme et les moyens offerts pour les atteindre. Ceux et celles qui désirent par la suite poursuivre leur démarche avec le programme sont accueillis par des consultants et consultantes en emploi qui font, avec eux, une évaluation de besoins. Cette évaluation se fait sous forme d'entrevues pendant lesquelles l'occasion leur est donnée d'exprimer les frustrations ressenties dans leur démarche vers l'indépendance. Grosso modo, les employés du programme chercheront à déterminer si leur client a un objectif de carrière à identifier, les étapes qui ont été franchies vers l'atteinte de cet objectif et à connaître sa perception des étapes qui restent à franchir et les sources d'appui dont il bénéficie.

Pour parvenir à son but, le programme l'Avenir est structuré en deux phases: la formation préparatoire à l'emploi qui peut être ou non subventionnée et le stage subventionné en milieu de travail. L'objectif du stage en milieu de travail est, évidemment, d'encourager les employeurs à fournir une expérience productive aux jeunes qui n'auraient pas accès à cette formation sans le soutien financier du programme. La responsabilité de dénicher ce stage revient aux jeunes et avant de signer une entente de placement, le consultant ou la consultante du programme doit s'assurer que le stage est conforme aux exigences d'un plan de formation que les jeunes définissent pendant la phase préparatoire.

En effet, chaque jeune qui veut participer à un stage subventionné élabore un plan de formation à partir des constatations de son évaluation de besoins. Ce plan identifie les étapes à franchir pour atteindre l'objectif de carrière et doit souvent inclure une coopération étroite avec certaines agences communautaires. Il est évident que les besoins de formation ne sont pas les mêmes pour 
une jeune diplômée du secondaire ou d'un collège communautaire, que pour un jeune décrocheur et le contenu d'un bon plan de formation doit tenir compte des besoins de chaque individu. Dans certain cas, la phase préparatoire est d'une courte durée; pour d'autres, elle sera plus complexe et il est même possible d'inclure plus d'un stage en milieu de travail dans l'élaboration du plan de formation.

\section{Histoire de deux jeunes participants}

Le cheminement de deux jeunes dans le cadre du programme l'Avenir permet de mieux apprécier les défis et les enjeux auxquels ils et elles sont confrontés et les ressources qu'ils apportent et qu'ils en retirent.

Un jeune homme de dix-sept ans a demandé l'aide du programme dans sa recherche d'emploi. Sa mère qui élevait seule cinq enfants dont il était l'aîné, avait insisté qu'il laisse l'école pour travailler alors qu'il n'avait complété que 16 crédits du secondaire. Quoiqu'il avait présenté ici et là des demandes d'emploi, il était au comble du désespoir lorsque nous avons fait sa connaissance. Il voulait travailler soit comme mineur, soit comme camionneur mais aucun employeur ne s'intéressait à lui. Il a été invité à compléter un stage subventionné de formation préparatoire lors duquel il s'est renseigné, en se servant des ressources du programme et en interviewant des employeurs, sur les éléments aptes à faciliter sa demande d'emploi. Il a découvert que l'industrie minière au Canada était en décroissance à l'époque et qu'il lui fallait un permis de conduite de classe A pour opérer un camion. Après avoir identifié où il pouvait recevoir la formation nécessaire pour réussir un examen du ministère du Transport en vue d'obtenir son permis, il a complété un stage de travail dans un entrepôt. Pendant son stage, non seulement a-t-il chargé des camions, il a fait de la route avec un camionneur d'expérience et a réussi à épargner la somme nécessaire pour débourser les frais d'inscription à son instruction. Il est arrêté nous saluer 3 ans après son stage. Il arrivait de Toronto en route vers Winnipeg, au volant d'un camion chargé de marchandise. 
Il arrive parfois que l'intervention d'autres agences communautaires soit nécessaire avant que le programme l'Avenir ne puisse agir. C'était le cas alors qu'une jeune femme âgée de 19 ans s'est présentée au programme pour discuter de sa situation avec une consultante. Elle déplorait les conditions dans lesquelles elle élevait son enfant de deux ans. Elle a d'abord été référée à une agence de soutien aux femmes, puisque le counselling offert au programme l'Avenir se limite à l'emploi. Lorsqu'elle est revenue au programme, c'était pour poursuivre son ambition de travailler dans le domaine de la comptabilité. Pendant une phase préparatoire à l'emploi non-subventionné, elle s'est familiarisée avec les critères d'admission comme apprenante adulte à un collège communautaire, elle a identifié des employeurs en mesure de lui fournir un stage de travail stimulant et elle a participé à un atelier en techniques de recherche d'emploi. Elle a signé une entente de participation avec le programme qui lui a permis à la fois de compléter les crédits du secondaire nécessaires à la réussite de ses études collégiales et de travailler avec une agence qui fait de la tenue de livre pour de petites entreprises. Six mois après avoir reçu un diplôme d'études collégiales en affaires avec concentration en comptabilité, elle était toujours sans emploi. Un placement subventionné par le programme lui a permis de décrocher un poste en comptabilité chez un entrepreneur.

\section{Participation au programme l'Avenir en Ontario français}

Depuis l'entrée en vigueur du programme, le nombre de jeunes francophones qui en bénéficie varie entre environ 1400 et 2000 à chaque année. Le tableau ci-dessous offre un aperçu du nombre de participants ayant identifié le français comme langue maternelle et le pourcentage de jeunes francophones par rapport au nombre total de participants. 
Tableau 1 *

Participation francophone au programme l'Avenir pour l'ensemble de la province

\begin{tabular}{lcc}
\hline ANNÉE & \multicolumn{2}{c}{ PROVINCIAL } \\
& $\#$ & $\mathbf{\%}$ \\
\hline $1998-89$ & 1876 & 8,3 \\
$1989-90$ & 1694 & 8,5 \\
$1990-91$ & 1527 & 7,0 \\
$1991-92$ & 1391 & 7,1 \\
$1992-93$ & 1642 & 7,1 \\
$1993-94$ & 1854 & 8,4 \\
$1994-95$ & 2052 & 8,7 \\
$1995-96$ & 1835 & 8,7 \\
\hline
\end{tabular}

\# Nombre de francophones

$\%$ Pourcentage du total de jeunes participant au programme

* source : ministère de l'Éducation et de la formation, Division de la préparation au milieu de travail.

\section{Accès au programme}

La disponibilité des services du programme l'Avenir en Ontario est assurée conjointement par les collèges communautaires et par les centres de consultation d'embauche pour les jeunes là où ils existent. Afin de connaître les coordonnées spécifiques d'un centre l'Avenir, les personnes intéressées sont priées de composer le 1-800-387-5656. 